\title{
Co-existence of chronic non-communicable diseases and common neoplasms among 2,462 endocrine adult inpatients - a retrospective analysis
}

\author{
Paweł Szychta ${ }^{1,3}$, Wojciech Szychta ${ }^{1,4}$, Andrzej Lewiński ${ }^{2,3}$, Małgorzata Karbownik-Lewińska ${ }^{1,3}$ \\ ${ }^{1}$ Department of Oncological Endocrinology, Medical University of Lodz, Poland \\ ${ }^{2}$ Department of Endocrinology and Metabolic Diseases, Medical University of Lodz, Poland \\ ${ }^{3}$ Polish Mother's Memorial Hospital \& Research Institute, Lodz, Poland \\ ${ }^{4} 1^{\text {st }}$ Chair and Department of Cardiology, Medical University of Warsaw, Poland
}

Szychta P, Szychta W, Lewiński A, Karbownik-Lewińska M. Co-existence of chronic non-communicable diseases and common neoplasms among 2,462 endocrine adult inpatients - a retrospective analysis. Ann Agric Environ Med. 2015; 22(4): 747-754. doi: 10.5604/12321966.1185788

\begin{abstract}
Objective. To analyze the coexistence of chronic non-communicable diseases (NCDs) and common neoplasms among endocrine adult inpatients.

Materials and method. The retrospective analysis was performed using clinical data of 2,462 adult patients (2,003 women and 459 men), hospitalized in the reference endocrine department. Diagnoses of 18 types of benign tumours and 16 types of malignant tumours, together with the most common 25 NCDs and demographic parameters, were all collected from the medical records. The most frequently found 6 types of benign tumours (of thyroid, pituitary, uterus, breast, adrenal and prostate) and 4 types of malignant tumours (of thyroid, breast, prostate and uterus) were taken for further statistical analyses. Results. Age predicted the existence of accumulated as well as individual types of benign and malignant tumours, whereas BMI predicted the occurrence of accumulated and some individual types of benign tumours. Accumulated as well as individual types of benign and malignant tumours coexisted more frequently with several NCDs, such as diabetes, hypertension, metabolic syndrome, osteoporosis, Graves' disease, coronary artery disease, state after cholecystectomy, thus being disorders usually resulting from excessive exposure to harmful environmental factors. The most distinct coexistence was found between breast cancer and metabolic syndrome, between breast cancer and Graves' disease, between cancer of the uterus and type 2 diabetes, between cancer of the uterus and metabolic syndrome, and between cancer of the uterus and dyslipidemia.

Conclusion. The results obtained indicate a significant relationship between the most common NCDs and several cancers in endocrine adult patients, which suggests that the prevention of the former may reduce the frequency of the latter.
\end{abstract}

\section{Key words}

cancer, tumour, malignant, benign, coexistence, environmental factors, chronic non-communicable diseases, endocrine diseases

\section{INTRODUCTION}

Carcinogenesis is associated with both genetic predisposition and exposure to environmental factors [1]. Currently, genetic factors are widely regarded as the most important in etiology of carcinogenesis, which may downplay the role of environmental factors. In fact, the latter could potentially constitute the major cause of the common cancers [2]. Environmental factors such as smoking, alcohol abuse, poor diet and lifestyle or infectious agents contribute to the development of a wide range of chronic, civilization-related diseases, usually called non-communicable diseases (NCDs). Therefore, some of the chronic diseases can act as markers of exposure to environmental factors which, in turn, might contribute to the development of cancer.

The WHO defines cancer as a disease which is largely preventable, especially by avoidance of harmful environmental

Address for correspondence: Małgorzata Karbownik-Lewińska, Department of Oncological Endocrinology, Medical University of Lodz, Zeligowski 7/9, 90-752 Lodz, Poland

E-mail: MKarbownik@hotmail.com

Received: 30 April 2014; accepted: 28 May 2014 factors [3]. As previously reported, migrants suffer from malignant tumours with a frequency corresponding to the general risk observed in the local population and independent from the original place of residence [4]. In monozygotic twins, environmental factors are responsible for the development of cervical cancer in $100 \%$, ovarian cancer and leukemia in $78 \%$, lung cancer in $74 \%$, breast cancer in $73 \%$ and prostate cancer in 58\% [5]. The incidence of cancers in middle-aged and elderly people could be reduced even by $80-90 \%$ by eliminating the harmful effects of environmental factors [4]. Healthy life-style supported by regulatory and legislative measures should include: healthy diet, physical activity, safe sex, breastfeeding, avoidance of tobacco use, rational alcohol habits and limited exposure to sunlight.

Some of the results of exposure to environmental factors disrupt the harmony of the endocrine environment. In physiological conditions, cell signaling pathways, involved in the regulation of cell metabolism, are under hormonal control, and their disruption promotes the initiation of carcinogenesis [6]. Endocrine diseases were considered risk factors for malignant neoplasms of the responsive organs, such as breast, ovary, endometrium, prostate, testes and 
thyroid [7]. Apart from responding to a wide range of hormones, some cancer cells secrete hormones and thus contribute to cancer progression [6]. It is suggested that the formation of some cancers can potentially be prevented by regulation of the hormonal milieu. Therefore, the relationship between NCDs and cancers can be assessed in the selected group of endocrine inpatients.

The aim of the study was a retrospective analysis of the coexistence of NCDs and commonly diagnosed neoplasms among endocrine adult inpatients.

\section{MATERIALS AND METHOD}

The retrospective audit was performed using the data of 2,462 adult patients (aged 38.95 \pm 17.02 years), hospitalized in the Department of Endocrinology and Metabolic Diseases at the Polish Mother's Memorial Hospital \& Research Institute in Lodz, Poland, within a 4-year period between 2002 and 2005 (Tab. 1).

Table 1. Distribution of the accumulated benign and malignant tumours in all adult patients and after stratification to gender

\begin{tabular}{|c|c|c|c|c|c|c|c|}
\hline \multirow{3}{*}{ Population } & \multirow[b]{3}{*}{ No. } & \multirow[b]{3}{*}{$\%$} & \multicolumn{4}{|c|}{ No. of tumours } & \\
\hline & & & \multicolumn{2}{|c|}{$\begin{array}{l}\text { Accumulated } \\
\text { benign }\end{array}$} & \multicolumn{2}{|c|}{$\begin{array}{l}\text { Accumulated } \\
\text { malignant }\end{array}$} & \\
\hline & & & No. & $\%$ & No. & $\%$ & \\
\hline All patients & 2,462 & 100 & 623 & 25.30 & 79 & 3.21 & $p_{2}<0.0001$ \\
\hline Females & 2,003 & 81.36 & 544 & 27.16 & 53 & 2.65 & $p_{2}=0.0001$ \\
\hline \multirow[t]{2}{*}{ Males } & 459 & 18.64 & 79 & 17.21 & 26 & 5.66 & $p_{2}=\mathrm{ns}$ \\
\hline & & & \multicolumn{2}{|c|}{$p_{1}<0.0001$} & \multicolumn{2}{|c|}{$p_{1}=0.0007$} & \\
\hline
\end{tabular}

Statistical evaluation was performed by the Ratio Comparison Test; $p$ - level of statistical significance $(p<0.05)$, ns - non-significant, $p_{1}$ - females vs. males, $p_{2}-$ accumulated benign tumours vs. accumulated malignant tumors
Diagnoses of the neoplasms and the most common disorders, together with demographic parameters, were collected by a computer programme designed by one of the authors (PS). The most common benign tumours were detected in thyroid, pituitary, uterus, breast and adrenal gland, whereas malignancies were found in thyroid, breast, prostate and uterus (Tab. 2). Coexisting NCDs included: obesity, diabetes overall (accumulated types of diabetes), type 2 diabetes, type 1 diabetes, gestational diabetes, polycystic ovary syndrome, hypertension, metabolic syndrome, hyperprolactinemia, osteopenia, osteoporosis, Graves' disease, coronary artery disease, hypothyroidism (independently of etiology), state after cholecystectomy, hirsutism, primary infertility, anorexia nervosa, hyperlipidemia, hyperthyroidism, Hashimoto disease, hyposomatotropinism, dyslipidemia, gastric ulcer, gynecomastia and atherosclerosis (Tab. 3). Relationships between NCDs and different types of tumors were analyzed in all patients (Tab. 4) and separately for women (Tab. 5) and men (Tab. 6). The analyzed demographic parameters included age and body mass index (BMI) (Tab. 7 and 8).

The data were statistically analysed using the two-sided ratio comparison test to evaluate the frequency of events. Univariate logistic regression analysis was used to determine which continuous variable might have predicted benign or malignant tumours. In order to adjust for several risk factors, multivariate logistic regression analysis was performed with all the variables found to be significant at the univariate analysis, entering in a single step. The $\chi 2$ test of independence was used to determine which dichotomized variable might have coexisted with benign or malignant tumours. The results are presented as mean \pm standard deviation (SD). Statistical significance was determined at the level of $p<0.05$.

Table 2. Distribution of particular types of benign and malignant tumours in all adult patients and after stratification to gender

\begin{tabular}{|c|c|c|c|c|c|c|c|c|c|c|c|c|c|}
\hline \multicolumn{7}{|c|}{ Benign tumors } & \multicolumn{7}{|c|}{ Malignant tumors } \\
\hline \multirow{2}{*}{ Location } & \multicolumn{2}{|c|}{ All patients } & \multicolumn{2}{|c|}{ Females } & \multicolumn{2}{|c|}{ Males } & \multirow{2}{*}{ Location } & \multicolumn{2}{|c|}{ All patients } & \multicolumn{2}{|c|}{ Females } & \multicolumn{2}{|c|}{ Males } \\
\hline & No. & $\%$ & No. & $\%$ & No. & $\%$ & & No. & $\%$ & No. & $\%$ & No. & $\%$ \\
\hline Nodular goiter & 179 & 7.27 & 153 & 7.63 & 26 & 5.66 & Thyroid gland & 24 & 0.97 & 18 & 0.90 & 6 & 1.31 \\
\hline Pituitary & 137 & 5.56 & 117 & 5.84 & 20 & 4.36 & Breast & 9 & 0.36 & 9 & 0.53 & & \\
\hline Uterus & 134 & 5.44 & 134 & 6.69 & & & Prostate & 8 & 0.32 & & & 8 & 1.75 \\
\hline Adrenal & 29 & 1.18 & 22 & 1.10 & 7 & 1.53 & Lung & 5 & 0.20 & & & 5 & 1.09 \\
\hline Ovary & 25 & 1.02 & 25 & 1.25 & & & Leukemia & 5 & 0.20 & 5 & 0.25 & & \\
\hline Prostate & 14 & 0.57 & & & 14 & 3.06 & Ovary & 4 & 0.16 & 4 & 0.20 & & \\
\hline Brain & 11 & 0.45 & 7 & 0.35 & 4 & 0.87 & Kidney & 4 & 0.16 & 1 & 0.05 & 3 & 0.66 \\
\hline Liver & 9 & 0.37 & 9 & 0.45 & & & Adrenal & 2 & 0.08 & 1 & 0.05 & 1 & 0.22 \\
\hline Kidney & 5 & 0.20 & 3 & 0.15 & 2 & 0.44 & Parathyroid gland & 2 & 0.08 & 2 & 0.10 & & \\
\hline Salivary gland & 4 & 0.16 & 3 & 0.15 & 1 & 0.22 & Brain & 1 & 0.04 & 1 & 0.05 & & \\
\hline Pineal gland & 2 & 0.08 & 2 & 0.10 & & & Liver & 1 & 0.04 & & & 1 & 0.22 \\
\hline Parathyroid gland & 2 & 0.08 & 2 & 0.10 & & & Skin & 1 & 0.04 & 1 & 0.05 & & \\
\hline Pancreas & 2 & 0.08 & 2 & 0.10 & & & Generalized malignancy & 1 & 0.04 & & & 1 & 0.22 \\
\hline Lung & 2 & 0.08 & 1 & 0.05 & 1 & 0.22 & Urinary bladder & 1 & 0.04 & 1 & 0.05 & & \\
\hline Urinary bladder & 1 & 0.04 & & & 1 & 0.22 & & & & & & & \\
\hline Gall bladder & 1 & 0.04 & & & 1 & 0.22 & & & & & & & \\
\hline
\end{tabular}


Table 3. Coexistence of commonly diagnosed NCDs with the most frequently found individual types of benign or malignant tumours in all adult patients and after stratification to gender

\begin{tabular}{|c|c|c|c|c|c|c|c|c|c|c|c|c|c|c|c|}
\hline \multicolumn{4}{|l|}{ Diagnosis } & \multicolumn{4}{|c|}{ Accumulated tumours in all patients } & \multicolumn{4}{|c|}{ Accumulated tumours in women } & \multicolumn{4}{|c|}{ Accumulated tumours in men } \\
\hline & & \multirow{2}{*}{\multicolumn{2}{|c|}{ No. of cases }} & \multicolumn{2}{|c|}{ Benign } & \multicolumn{2}{|c|}{ Malignant } & \multicolumn{2}{|c|}{ Benign } & \multicolumn{2}{|c|}{ Malignant } & \multicolumn{2}{|c|}{ Benign } & \multicolumn{2}{|c|}{ Malignant } \\
\hline & & & & $\chi^{2}$ & $p$ & $\chi^{2}$ & $p$ & $\chi^{2}$ & $p$ & $\chi^{2}$ & $p$ & $\chi^{2}$ & $p$ & $\chi^{2}$ & $p$ \\
\hline Obesity & & & 516 & 1.62 & ns & 1.71 & ns & 1.64 & ns & 0.21 & ns & 0.29 & ns & 3.16 & ns \\
\hline \multirow{4}{*}{ Diabetes } & All types & & 383 & 2.38 & ns & 0.24 & ns & 0.96 & ns & 0.09 & ns & 0.42 & ns & 0.87 & ns \\
\hline & Type 2 & \multirow{3}{*}{383} & 239 & 2.46 & ns & 0.52 & ns & 4.61 & 0.0317 & 1.38 & ns & 0.27 & ns & 0.62 & ns \\
\hline & Type 1 & & 110 & 13.30 & 0.0002 & 1.73 & ns & 8.99 & 0.0027 & 2.23 & ns & 3.89 & 0.0484 & 0.12 & ns \\
\hline & Gestational & & 34 & & & & & 5.68 & 0.0171 & 0.87 & ns & & & & \\
\hline \multicolumn{2}{|c|}{ Polycystic ovary syndrome } & & 339 & & & & & 70.40 & 0.0000 & 8.33 & 0.0038 & & & & \\
\hline \multicolumn{2}{|c|}{ Hypertension } & & 296 & 66.65 & 0.0000 & 16.23 & 0.0001 & 63.36 & 0.0000 & 7.03 & 0.0080 & 11.62 & 0.0006 & 6.22 & 0.0126 \\
\hline \multicolumn{2}{|c|}{ Metabolic syndrome } & & 260 & 4.80 & 0.0284 & 0.20 & ns & 10.99 & 0.0009 & 1.22 & ns & 0.84 & ns & 0.98 & ns \\
\hline \multicolumn{2}{|c|}{ Hyperprolactinemia } & & 215 & 0.26 & ns & 2.09 & ns & 1.72 & ns & 1.01 & ns & 4.23 & 0.0394 & 0.70 & ns \\
\hline \multicolumn{2}{|c|}{ Osteoporosis } & & 177 & 34.72 & 0.0000 & 0.09 & ns & 28.42 & 0.0000 & 0.03 & ns & 6.48 & 0.0108 & 0.08 & ns \\
\hline \multicolumn{2}{|c|}{ Graves' disease } & & 136 & 3.94 & 0.0469 & 0.22 & ns & 3.32 & ns & 1.35 & ns & 1.39 & ns & 0.87 & ns \\
\hline \multicolumn{2}{|c|}{ Coronary artery disease } & & 135 & 11.33 & 0.0007 & 9.49 & 0.0020 & 10.96 & 0.0009 & 0.13 & ns & 2.12 & ns & 14.98 & 0.0001 \\
\hline \multicolumn{2}{|c|}{ Hypothyroidism } & & 118 & 9.54 & 0.0020 & 1.83 & ns & 7.89 & 0.0049 & 2.33 & ns & 0.97 & ns & 0.08 & ns \\
\hline \multicolumn{2}{|c|}{ State after cholecystectomy } & & 105 & 5.22 & 0.0223 & 8.00 & 0.0046 & 8.44 & 0.0036 & 3.64 & 0.0561 & 1.59 & ns & 5.92 & 0.0149 \\
\hline \multicolumn{2}{|l|}{ Hirsutism } & & 72 & & & & & 0.68 & ns & 0.01 & ns & & & & \\
\hline \multicolumn{2}{|c|}{ Primary infertility } & & 61 & & & & & 9.46 & 0.0020 & 1.58 & ns & & & & \\
\hline \multicolumn{2}{|c|}{ Anorexia nervosa } & & 39 & & & & & 6.87 & 0.0087 & 0.98 & ns & & & & \\
\hline \multicolumn{2}{|c|}{ Hyperlipidemia } & & 37 & 3.54 & ns & 0.01 & ns & 5.15 & 0.0231 & 0.30 & ns & 0.18 & ns & 0.76 & ns \\
\hline Hyperthyr & & & 37 & 0.25 & ns & 1.16 & ns & 0.04 & ns & 0.60 & ns & 0.08 & ns & 0.76 & ns \\
\hline Hashimotc & ease & & 32 & 0.13 & ns & 0.001 & ns & 0.08 & ns & 0.14 & ns & 0.02 & ns & 0.26 & ns \\
\hline Gastric ulc & & & 19 & 0.94 & ns & 3.71 & ns & 2.28 & ns & 7.08 & 0.0077 & 0.83 & ns & 0.21 & ns \\
\hline Gynecom & & & 17 & 1.10 & ns & 0.53 & ns & & & & & 1.21 & ns & 0.81 & ns \\
\hline Atheroscle & & & 14 & 3.41 & ns & 0.82 & ns & 0.87 & ns & 3.20 & ns & 4.58 & 0.0322 & 0.32 & ns \\
\hline
\end{tabular}

Statistical evaluation performed by $\chi^{2}$ test. $p$ - level of statistical significance $(p<0.05)$; ns - non-significant

\section{RESULTS}

Of the 2,462 adult endocrine patients enrolled into the study, 2,003 (81.36\%) were women and 459 (18.64\%) were men. 550 patients had benign tumor(s) and 74 patients had malignant tumour(s); the total number of all benign tumours was 623 and the total number of all malignant tumours was 79 (Tab. 1). Benign tumours occurred more frequently than malignant tumours, either in the whole group of patients or in females. Benign tumours occurred more frequently in women $(\mathrm{p}<0.0001)$, whereas malignant tumours occurred more frequently in men $(\mathrm{p}=0.0007)$ (Tab. 1$)$.

The distribution of individual types of tumours was analyzed. The most frequently diagnosed benign tumours were found in the thyroid (nodular goiter), followed by pituitary (adenomas), uterus (mainly uterine myomas), breast, adrenal, ovary and prostate. The most frequent malignancies were: thyroid cancer, followed by cancer of the breast, prostate, uterus, lung, ovary and kidney, and leukemia (Tab. 2).

The selected NCDs were analyzed for their coexistence with accumulated benign and malignant tumours (Tab. 3). In patients of both genders, the accumulated benign tumours coexisted more frequently with: type 1 diabetes, hypertension, metabolic syndrome, osteoporosis, Graves' disease, coronary artery disease, hypothyroidism and state after cholecystectomy, whereas the accumulated malignant tumours were observed more commonly with: hypertension, coronary artery disease and state after cholecystectomy.

In women, accumulated benign tumours coexisted more often with: type 2 diabetes, type 1 diabetes, gestational diabetes, polycystic ovary syndrome, hypertension, metabolic syndrome, osteoporosis, coronary artery disease, hypothyroidism, state after cholecystectomy, primary infertility, anorexia nervosa and hyperlipidemia, whereas accumulated malignant tumours were found more frequently with: polycystic ovary syndrome, hypertension, state after cholecystectomy and gastric ulcer. In men, accumulated benign tumours were seen more frequently with: type 1 diabetes, hypertension, hyperprolactinemia, osteoporosis and atherosclerosis, whereas accumulated malignant tumours were diagnosed more commonly with: hypertension, coronary artery disease and state after cholecystectomy (Tab. 3).

The relationship between NCDs with the individual types of benign and malignant tumours among all patients is presented in Table 4. Nodular goiter coexisted more frequently with: type 
Table 4. Coexistence of different disorders with the most frequently diagnosed individual types of benign or malignant tumors in all adult patients

\begin{tabular}{|c|c|c|c|c|c|c|c|c|c|}
\hline & & & & & & & & Malig & nors \\
\hline & hosis & & & & & & & & \\
\hline & & $\chi^{2}$ & $p$ & $\chi^{2}$ & $p$ & $\chi^{2}$ & $p$ & $\chi^{2}$ & $p$ \\
\hline Obesity & & 0.01 & ns & 0.24 & ns & 13.21 & 0.0002 & 0.98 & ns \\
\hline & All types & 1.73 & ns & 13.79 & 0.0002 & 0.06 & ns & 0.17 & ns \\
\hline abotact & Type 2 & 10.95 & 0.0009 & 6.07 & 0.0137 & 1.90 & ns & 0.05 & ns \\
\hline Didactes & Type 1 & 3.52 & ns & 6.78 & 0.0091 & 1.37 & ns & 0.01 & ns \\
\hline & Gestational & 0.95 & ns & 0.45 & ns & 0.41 & ns & 0.33 & ns \\
\hline Hypertens & & 56.44 & 0.0000 & 0.17 & ns & 10.02 & 0.0015 & 10.40 & 0.0013 \\
\hline Metabolic & trome & 2.37 & ns & 4.56 & 0.0326 & 0.32 & ns & 2.86 & ns \\
\hline Hyperprol & emia & 8.54 & 0.0034 & 16.48 & 0.0001 & 0.12 & ns & 0.01 & ns \\
\hline Osteopeni & & 5.88 & 0.0152 & 0.53 & ns & 1.04 & ns & 0.59 & ns \\
\hline Osteoporo & & 20.67 & 0.0001 & 0.08 & ns & 0.43 & ns & 0.33 & ns \\
\hline Graves' dis & & 4.00 & 0.0454 & 6.38 & 0.0114 & 0.24 & ns & 1.41 & ns \\
\hline Coronary a & $y$ disease & 12.05 & 0.0005 & 3.03 & ns & 1.33 & ns & 11.01 & 0.0009 \\
\hline Hypothyro & & 7.27 & 0.0070 & 0.34 & ns & 0.28 & ns & 3.15 & ns \\
\hline State after & lecystectomy & 4.24 & 0.0392 & 1.52 & ns & 2.65 & ns & 0.98 & ns \\
\hline Hyperlipid & & 4.45 & 0.0347 & 0.46 & ns & 0.75 & ns & 1.16 & ns \\
\hline Hyperthyr & & 0.69 & ns & 2.21 & ns & 0.44 & $\mathrm{~ns}$ & 0.36 & ns \\
\hline Hashimoto & ease & 0.82 & ns & 1.91 & ns & 0.38 & ns & 0.31 & ns \\
\hline Hyposoma & opinism & 1.98 & ns & 0.28 & ns & 0.30 & ns & 0.24 & ns \\
\hline Dyslipiden & & 1.50 & ns & 0.89 & ns & 0.22 & ns & 0.18 & ns \\
\hline Gastric ulc & & 0.30 & ns & 1.12 & ns & 0.22 & $\mathrm{~ns}$ & 3.64 & 0.0562 \\
\hline Atheroscle & & 4.18 & 0.0407 & 0.06 & ns & 0.16 & ns & 0.13 & ns \\
\hline
\end{tabular}

Statistical evaluation performed by $\chi^{2}$ test. $p$ - level of statistical significance $(p<0.05)$, ns - non-significant

2 diabetes, hypertension, hyperprolactinemia, osteopenia, osteoporosis, Graves' disease, coronary artery disease, hypothyroidism, state after cholecystectomy, hyperlipidemia and atherosclerosis. Benign tumours of pituitary coexisted more frequently with: diabetes in overall, type 2 diabetes, type 1 diabetes, metabolic syndrome, hyperprolactinemia and Graves' disease. Adrenal gland benign tumours occurred more frequently in patients suffering from: obesity and hypertension. In relation to malignant tumours observed in patients of both genders, the only diagnosed cancer of thyroid coexisted more frequently with hypertension, coronary artery disease and gastric ulcer (Tab. 4).

When statistical evaluation was performed after stratification to gender, different results were obtained concerning the coexistence of NCDs with particular benign and malignant tumours (Tab. 5 and 6).

In female patients, nodular goiter coexisted more frequently with: diabetes in overall, type 2 diabetes, polycystic ovary syndrome, hypertension, metabolic syndrome, hyperprolactinemia, osteopenia, osteoporosis, coronary artery disease, state after cholecystectomy, hirsutism and hyperlipidemia. Benign tumours of pituitary coexisted more frequently with: diabetes in overall, type 1 diabetes, polycystic ovary syndrome, hyperprolactinemia, Graves' disease, hyposomatotropinism and dyslipidemia. Benign tumours of uterus coexisted more frequently with: type 2 diabetes, type 1 diabetes, polycystic ovary syndrome, hypertension, metabolic syndrome, osteoporosis, coronary artery disease, hirsutism, Hashimoto disease and gastric ulcer. Breast benign tumours coexisted more frequently with: polycystic ovary syndrome, metabolic syndrome and coronary artery disease.
Benign tumours of adrenal gland occurred more frequently with: obesity, polycystic ovary syndrome, hypertension and state after cholecystectomy (Tab. 5).

In women, thyroid cancer coexisted more frequently with: hypothyroidism and gastric ulcer. Breast cancer coexisted more frequently with: metabolic syndrome, Graves' disease, state after cholecystectomy and atherosclerosis. Uterus cancer coexisted more frequently with type 2 diabetes, metabolic syndrome and dyslipidemia (Tab. 5).

In turn, in male patients, nodular goiter coexisted more frequently with hypertension and hypothyroidism. Benign tumours of pituitary coexisted more frequently with: diabetes overall and hyperprolactinemia. Benign tumours of adrenal gland coexisted more frequently with obesity. Benign tumours of prostate coexisted more frequently with: diabetes overall, type 2 diabetes, hypertension, osteoporosis and atherosclerosis (Tab. 6).

In relation to individual types of malignancies analyzed in men, thyroid cancer coexisted more frequently with hypertension and coronary artery disease. Prostate cancer coexisted more frequently with hypertension, osteoporosis and coronary artery disease (Tab. 6).

It was also evaluated whether continuous variables, such as age and BMI, might have predicted the existence of accumulated neoplasms (Tab. 7) or individual types of cancers (Tab. 8). Age predicted the existence of accumulated benign tumours and accumulated malignant tumours in all patients, and also separately among women and men. Following multivariate regression analysis, age constituted the only independent predictor of the existence of accumulated benign tumours in all patients and in women. In relation to 
Table 5. Coexistence of the commonly diagnosed NCDs with the most frequently found individual types of benign or malignant tumours in female adult patients

\begin{tabular}{|c|c|c|c|c|c|c|c|c|c|c|c|c|c|c|c|c|c|}
\hline \multirow{3}{*}{\multicolumn{2}{|c|}{ Diagnosis }} & \multicolumn{10}{|c|}{ Benign tumors } & \multicolumn{6}{|c|}{ Malignant tumors } \\
\hline & & \multicolumn{2}{|c|}{ Nodular goiter } & \multicolumn{2}{|c|}{ Pituitary } & \multicolumn{2}{|c|}{ Uterus } & \multicolumn{2}{|c|}{ Breast } & \multicolumn{2}{|c|}{ Adrenal } & \multicolumn{2}{|c|}{ Thyroid gland } & \multicolumn{2}{|c|}{ Breast } & \multicolumn{2}{|c|}{ Uterus } \\
\hline & & $\chi^{2}$ & $p$ & $\chi^{2}$ & $p$ & $\chi^{2}$ & $p$ & $\chi^{2}$ & $p$ & $\chi^{2}$ & $p$ & $\chi^{2}$ & $p$ & $\chi^{2}$ & $p$ & $\chi^{2}$ & $p$ \\
\hline Obesity & & 0.04 & ns & 0.02 & ns & 0.82 & ns & 0.01 & ns & 5.87 & 0.0154 & 0.63 & ns & 0.85 & ns & 0.04 & ns \\
\hline \multirow{3}{*}{ Diabetes } & All types & 4.74 & 0.0295 & 8.68 & 0.0032 & 0.02 & ns & 0.02 & ns & 0.33 & ns & 0.07 & ns & 0.16 & ns & 2.12 & ns \\
\hline & Type 2 & 18.76 & 0.0000 & 2.97 & ns & 4.25 & 0.0393 & 0.86 & ns & 0.08 & ns & 0.34 & ns & 0.04 & ns & 5.80 & 0.0159 \\
\hline & Gestational & 1.00 & ns & 0.48 & ns & 2.38 & ns & 0.96 & ns & 0.37 & ns & 0.30 & ns & 0.18 & ns & 0.10 & ns \\
\hline \multicolumn{2}{|c|}{ Polycystic ovary syndrome } & 31.17 & 0.0000 & 5.00 & 0.0253 & 19.61 & 0.0000 & 7.30 & 0.0060 & 4.52 & 0.0333 & 1.66 & ns & 2.25 & ns & 1.22 & ns \\
\hline \multicolumn{2}{|c|}{ Hypertension } & 60.15 & 0.0000 & 0.07 & ns & 19.99 & 0.0000 & 3.46 & ns & 10.99 & 0.0009 & 2.73 & ns & 3.39 & ns & 3.40 & ns \\
\hline \multicolumn{2}{|c|}{ Metabolic syndrome } & 3.88 & 0.0487 & 2.55 & ns & 23.44 & 0.0000 & 5.03 & 0.0249 & 0.01 & ns & 1.85 & ns & 4.25 & 0.0392 & 4.13 & 0.0420 \\
\hline \multicolumn{2}{|c|}{ Hyperprolactinemia } & 8.48 & 0.0036 & 12.55 & 0.0003 & 1.72 & ns & 0.54 & ns & 0.75 & ns & 0.02 & ns & 0.01 & ns & 0.67 & ns \\
\hline \multicolumn{2}{|c|}{ Osteoporosis } & 17.95 & 0.0000 & 0.79 & ns & 5.01 & 0.0252 & 2.44 & ns & 1.38 & ns & 0.07 & ns & 0.06 & ns & 0.81 & ns \\
\hline \multicolumn{2}{|c|}{ Graves' disease } & 3.35 & ns & 5.82 & 0.0158 & 0.59 & ns & 2.33 & ns & 0.08 & ns & 1.15 & ns & 9.41 & 0.0021 & 0.38 & ns \\
\hline \multicolumn{2}{|c|}{ Coronary artery disease } & 14.51 & 0.0001 & 1.35 & ns & 3.78 & 0.0520 & 7.50 & 0.0061 & 0.90 & ns & 1.59 & ns & 0.55 & ns & 0.30 & ns \\
\hline \multicolumn{2}{|c|}{ Hypothyroidism } & 2.48 & ns & 0.73 & ns & 0.01 & ns & 1.69 & ns & 0.71 & ns & 4.95 & 0.0261 & 0.59 & ns & 0.32 & ns \\
\hline \multicolumn{2}{|c|}{$\begin{array}{l}\text { State after } \\
\text { cholecystectomy }\end{array}$} & 4.70 & 0.0301 & 0.98 & ns & 0.25 & ns & 2.82 & ns & 4.52 & 0.0333 & 1.95 & ns & 5.01 & 0.0252 & 2.15 & ns \\
\hline \multicolumn{2}{|l|}{ Hirsutism } & 4.13 & 0.0421 & 0.38 & ns & 4.14 & 0.0418 & 0.54 & ns & 0.82 & ns & 0.20 & ns & 0.96 & ns & 0.22 & ns \\
\hline \multicolumn{2}{|c|}{ Primary infertility } & 3.04 & ns & 1.90 & ns & 2.39 & ns & 1.74 & ns & 0.67 & ns & 0.55 & ns & 0.33 & ns & 0.18 & ns \\
\hline \multicolumn{2}{|c|}{ Anorexia nervosa } & 3.12 & ns & 0.01 & ns & 2.68 & ns & 1.08 & ns & 0.41 & ns & 0.34 & ns & 0.20 & ns & 0.11 & ns \\
\hline \multicolumn{2}{|c|}{ Hyperlipidemia } & 6.56 & 0.0104 & 0.09 & ns & 0.19 & ns & 0.66 & ns & 2.26 & ns & 3.11 & ns & 0.12 & ns & 0.06 & ns \\
\hline Hyperthyr & sm & 0.03 & ns & 1.44 & ns & 1.54 & ns & 2.98 & ns & 0.25 & ns & 0.21 & ns & 0.12 & ns & 0.06 & ns \\
\hline Hashimotc & sease & 2.26 & ns & 1.69 & ns & 6.23 & 0.0125 & 0.08 & ns & 0.30 & ns & 0.24 & ns & 0.15 & ns & 0.08 & ns \\
\hline Gastric ulc & & 0.69 & ns & 0.93 & ns & 4.35 & 0.0369 & 0.83 & ns & 0.16 & ns & 5.65 & 0.0175 & 0.08 & ns & 0.04 & ns \\
\hline Atheroscle & & 3.43 & ns & 0.64 & ns & 0.57 & ns & 0.23 & ns & 0.08 & ns & 0.07 & ns & 21.02 & 0.0000 & 0.02 & ns \\
\hline
\end{tabular}

Statistical evaluation performed by $\chi^{2}$ test. $p$ - level of statistical significance $(p<0.05)$; ns - non-significant

the individual types of tumours, age predicted the higher occurrence of benign tumours (of thyroid, uterus, breast and adrenal gland) and of the three selected malignant tumours (thyroid, breast and prostate). Age also constituted the only independent predictor of the existence of benign tumours of thyroid and uterus. Increasing BMI predicted the occurrence of accumulated benign tumours in all patients and in women, and also determined the occurrence of nodular goiter and benign tumours of the uterus.

\section{DISCUSSION}

It is well known that the incidence of cancer as well as of other NCDs increases gradually with age. The previous report determined the annual risk of developing cancer at age of 20 years at the level of 1/10,000, at the age of $50-1 / 1,000$ and at the age of $80-1 / 100$ [1]. The incidence of malignant tumours, as it increases with the age, may be the result of gradual accumulation of mutations and epigenetic modifications, oxidative damage and modification of the immune system [8]. In the presented study, increasing age determined the higher occurrence of the three analyzed cancers, i.e. thyroid, breast and prostate.
Obesity is one of the most prevalent disorders in developed countries. Regulatory function was found for many environmental factors, including diet [9]. The relationship between obesity and cancer risk is currently being widely investigated. Interestingly, obesity was found to be an independent determinant for oxidative stress [10]. As previously described, there was an increasing trend of deaths from almost all analyzed cancers in the general population, proportionally to increasing BMI [11]. In contrast, in the current study, no relationship was observed between BMI and the risk of cancer among analyzed patients hospitalized for endocrine diseases.

Breast cancer is the most common malignancy for women worldwide. Based on recent research, social and environmental factors may play a more important role in the severity of the risk of breast cancer $(73 \%)$ than genetic factors (27\%), in contrast to the earlier hypothesis [12]. The importance of environmental factors is visible even in women with inherited mutations, such as BRCA1/2, and the lack of exercise, diet and the resulting obesity determined the higher occurrence of cancer [12]. The number of cases of breast cancer increased significantly in Poland since the 1990s. The gradual change of life-style is blamed for the above trend, including an unhealthy diet [1]. Previous 
Table 6. Coexistence of commonly diagnosed NCDs with the most frequently found individual types of benign or malignant tumors in male adult patients

\begin{tabular}{|c|c|c|c|c|c|c|c|c|c|c|c|c|c|}
\hline \multirow{3}{*}{\multicolumn{2}{|c|}{ Diagnosis }} & \multicolumn{8}{|c|}{ Benign tumors } & \multicolumn{4}{|c|}{ Malignant tumors } \\
\hline & & \multicolumn{2}{|c|}{ Nodular goiter } & \multicolumn{2}{|c|}{ Pituitary } & \multicolumn{2}{|c|}{ Adrenal } & \multicolumn{2}{|c|}{ Prostate } & \multicolumn{2}{|c|}{ Thyroid gland } & \multicolumn{2}{|c|}{ Prostate } \\
\hline & & $\chi^{2}$ & $p$ & $\chi^{2}$ & $p$ & $\chi^{2}$ & $p$ & $\chi^{2}$ & $p$ & $\chi^{2}$ & $p$ & $\chi^{2}$ & $p$ \\
\hline Obesity & & 0.01 & ns & 2.85 & ns & 8.75 & 0.0030 & 2.25 & ns & 0.28 & ns & 2.24 & ns \\
\hline \multirow[b]{2}{*}{ Diabetes } & All types & 0.61 & ns & 4.69 & 0.0302 & 1.08 & ns & 4.43 & 0.0352 & 0.26 & ns & 0.02 & ns \\
\hline & Type 2 & 0.01 & ns & 2.77 & ns & 2.49 & ns & 8.61 & 0.0033 & 1.46 & ns & 0.37 & ns \\
\hline \multicolumn{2}{|c|}{ Hypertension } & 4.21 & 0.0401 & 0.45 & ns & 0.40 & ns & 5.20 & 0.0226 & 8.81 & 0.0030 & 6.58 & 0.0103 \\
\hline \multicolumn{2}{|c|}{ Metabolic syndrome } & 0.01 & ns & 1.91 & ns & 0.80 & ns & 0.03 & ns & 1.17 & ns & 0.80 & ns \\
\hline \multicolumn{2}{|c|}{ Hyperprolactinemia } & 0.80 & ns & 3.90 & 0.0481 & 3.37 & ns & 0.42 & ns & 0.17 & ns & 0.20 & ns \\
\hline \multicolumn{2}{|c|}{ Osteopenia } & 0.56 & ns & 0.16 & ns & 0.44 & ns & 0.01 & ns & 0.70 & ns & 0.44 & ns \\
\hline \multicolumn{2}{|c|}{ Osteoporosis } & 2.75 & ns & 1.91 & ns & 0.55 & ns & 9.86 & 0.0017 & 0.47 & ns & 4.85 & 0.0276 \\
\hline \multicolumn{2}{|c|}{ Graves' disease } & 0.99 & ns & 0.75 & ns & 0.25 & ns & 0.57 & ns & 0.22 & ns & 0.25 & ns \\
\hline \multicolumn{2}{|c|}{ Hypothyroidism } & 12.76 & 0.0004 & 0.70 & ns & 0.24 & ns & 0.48 & ns & 0.20 & ns & 0.24 & ns \\
\hline \multicolumn{2}{|c|}{ State after cholecystectomy } & 0.01 & ns & 0.80 & ns & 0.27 & ns & 0.55 & ns & 0.23 & ns & 2.22 & ns \\
\hline \multicolumn{2}{|c|}{ Hyperlipidemia } & 0.05 & ns & 3.41 & ns & 0.22 & ns & 0.45 & ns & 0.19 & ns & 0.22 & ns \\
\hline \multicolumn{2}{|c|}{ Hyperthyreosis } & 1.99 & ns & 0.65 & ns & 0.22 & ns & 0.45 & ns & 0.19 & ns & 0.22 & ns \\
\hline \multicolumn{2}{|c|}{ Hashimoto disease } & 1.93 & ns & 0.23 & ns & 0.07 & ns & 0.15 & ns & 0.06 & ns & 0.07 & ns \\
\hline \multicolumn{2}{|c|}{ Hyposomatotropinism } & 1.39 & ns & 0.01 & ns & 0.35 & ns & 0.72 & ns & 0.30 & ns & 0.35 & ns \\
\hline \multicolumn{2}{|c|}{ Dyslipidemia } & 0.55 & ns & 0.41 & ns & 0.14 & ns & 0.28 & ns & 0.12 & ns & 0.14 & ns \\
\hline \multicolumn{2}{|c|}{ Gastric ulcer } & 0.24 & ns & 0.18 & ns & 0.06 & ns & 0.12 & ns & 0.05 & ns & 0.06 & ns \\
\hline \multicolumn{2}{|c|}{ Gynaecomastia } & 0.93 & ns & 0.19 & ns & 0.24 & ns & 0.48 & ns & 0.20 & ns & 0.24 & ns \\
\hline \multicolumn{2}{|c|}{ Atherosclerosis } & 1.37 & ns & 0.27 & ns & 0.09 & ns & 3.80 & 0.0513 & 0.08 & ns & 0.09 & ns \\
\hline
\end{tabular}

Statistical evaluation performed by $\chi^{2}$ test. $p$ - level of statistical significance $(p<0.05)$; ns - non-significant

reports gave contrary results for the relationship between obesity and breast cancer: in three separate studies, obesity was associated with a decreased incidence of breast cancer in premenopausal women, while the reverse was true for postmenopausal women [13].

Although in the current study no coexistence was found between obesity and breast cancer, it was observed that several diseases, caused partially by improper diet - such as atherosclerosis, metabolic syndrome and state after cholecystectomy - contributed to the increased ratio of breast cancer. Nor was any relationship found between diabetes or hypertension and breast cancer. Similarly, the previous report also found no significant association between diabetes, hypertension and breast cancer in women under 55 years of age [14]. Another study reported contrary results, where diabetes was associated with an increased ratio of breast cancer, with the relative risk $\mathrm{RR}=1.27$ (95\% CI 1.11-1.45) [15].

In a previous analysis of the selfsame series of women, the authors of the current study found that TSH (thyroidstimulating hormone) receptor antibodies (anty-TSHR) had a predictive value for breast cancer [16]. The presented study consequently reports a higher incidence of breast cancer in patients with Graves' disease.

Interestingly, hypothyroidism (treated or untreated) was previously reported to be associated with a lesser incidence of breast cancer [17]. In the current study, no relationship was observed between hypothyroidism or diagnosed Hashimoto disease and breast cancer.

The thyroid gland is involved in numerous metabolic pathways, and is therefore exposed to several intrinsic and extrinsic factors which could contribute to carcinogenesis.
Thus, thyroid neoplasms coexist with several chronic diseases in endocrine patients, as reported before and also by the authors of the presented study. A higher coexistence was previously reported between hypertension and thyroid cancer [18], which was confirmed by the research of the authors of this study. Noticeably, a higher coexistence was found between coronary artery disease and thyroid cancer. The above association was also previously reported, together with several triggering factors, such as hypertension, diabetes or cigarette smoking [19]. Invasive treatment of coronary artery disease, with low exposure to radiation during coronary angioplasty, is considered to have no significant influence on thyroid carcinogenesis [20]. Additionally, in the current study, patients with thyroid cancer were diagnosed more often also for gastric ulcer - a finding that requires further research.

Although Hashimoto disease was described as a risk factor for papillary thyroid cancer, in the current study Hashimoto disease and thyroid cancer did not occur more frequently in the same patients $[21,22]$.

Malignancies of uterus include cervical cancer and endometrial cancer, which constitute separate entities. In the previous reports, cervical cancer developed due to the human papillomavirus (HPV) infection, with the increased incidence among smoking women with lower socio-economic status [23]. In turn, endometrial cancer can be initiated by a distorted level of hormones and other metabolically active substances, such as adipokines, on the hormonally sensitive tissues lining the uterus. Previous research demonstrated a link between the metabolic syndrome, obesity and endometrial cancer, suggesting the role of leptin in the 

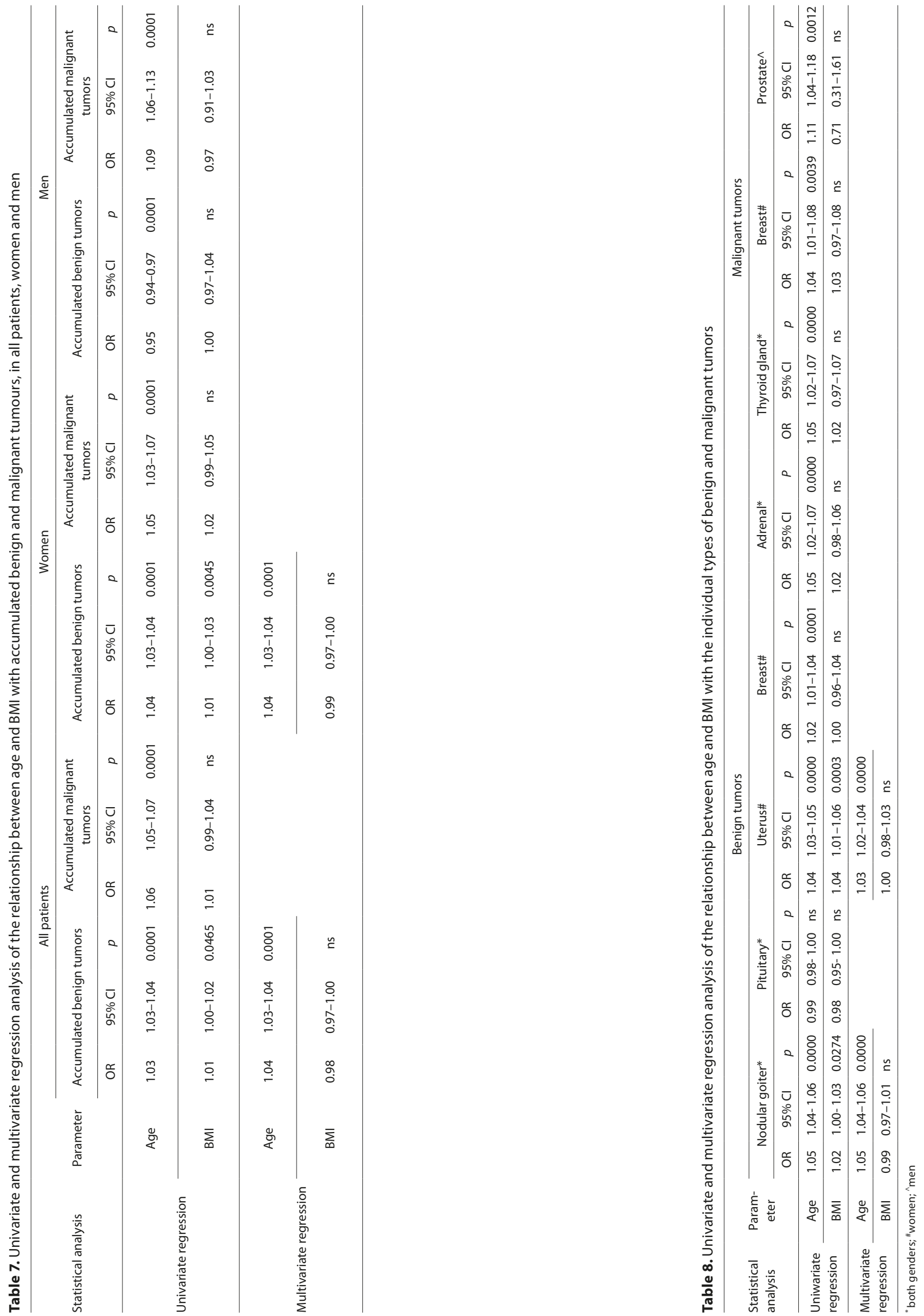
tumour growth [24]. Similar to the previous reports, the present study shows a higher coexistence of uterus cancer with dyslipidemia, metabolic syndrome and type 2 diabetes.

The primary cause of prostate cancer is unknown, and the reported risk factors are limited to age, race, familial predisposition, fat and red meat intake [25]. The protective factor is a regular diet rich in fruits and vegetables [1]. The significant geographic differences in the prevalence of prostate cancer suggest the important role of environmental factors in its etiology [1]. In the presented study, a more frequent coexistence was found with prostate cancer and such diseases as osteoporosis, hypertension and coronary artery disease, all of which were observed more frequently in older age, similar to the analyzed malignancy. However, in the presented study, no coexistence was found between prostate cancer and obesity; the relationship between obesity and the risk of prostate cancer is ambiguous, which has been stated in large cohort studies [25].

From the epidemiological and pathophysiological point of view, the clinical significance of the obtained results is the most convincing coexistence found between breast cancer and metabolic syndrome, between breast cancer and Graves' disease, between uterus cancer and type 2 diabetes, between uterus cancer and metabolic syndrome, and between uterus cancer and dyslipidemia.

It is difficult to evaluate to what extent the observations described in the presented endocrine inpatients differ from those in the general population, as the latter were not clearly established in the literature.

The limitations of this study include the retrospective type of analysis performed in one specific point of time, and in one reference endocrine department. Ratios of NCDs and neoplasms differed from the general population, which was probably associated with the profile of the hospitalized patients. Moreover, patients with both newly diagnosed tumors and cancers after oncological treatment were assessed, together with individuals suffering from recently found or treated NCDs. Therefore, coexistences could be depicted, but not cause-and-effect relationships among cancers, other NCDs, and oncological treatment. Indeed, exposure to cancer and its treatments, including chemotherapy and radiotherapy, may result in long-term adverse effects, including endocrine dysfunction. Another limitation of this study is the lack of data on lifestyle characteristics, such as smoking and drinking habits, which may interfere with NCDs.

Apart from the above limitations, the study produces clinically useful, epidemiological data on the coexistence of chronic diseases caused mostly by the civilization-related factors and common malignancies, which allow to consider effective, long-term, oncological prevention.

\section{CONCLUSIONS}

In conclusion, the presented findings indicate a significant relationship between the most common NCDs and several cancers in endocrine adult patients, which suggests that the prevention of the former may reduce the frequency of the latter.

\section{Acknowledgements}

The study was supported by the statutory funds No. 503/1107-03/503-11-001 from the Medical University of Lodz.

\section{REFERENCES}

1. Stare SM, Jozefowicz JJ. The Effects of Environmental Factors on Cancer Prevalence Rates and Specific Cancer Mortality Rates in a Sample of OECD Developed Countries. Int J Appl Eco. 2008; 5(2): 92-115.

2. Thompson PA. Counterpoint: genetic risk feedback for common disease time to test the waters. Cancer Epidemiol Biomarkers Prev. 2007; 16(9): 1727-1729.

3. Dauvergne, P. Cancer and Global Environmental Politics: Proposing A New Research Agenda. Global Environ Polit. 2005; 5(3): 6-13.

4. Doll R. Epidemiological evidence of the effects of behaviour and the environment on the risk of human cancer. Recent Results Cancer Res. 1998; 154: 3-21.

5. Lichtenstein P, Holm NV, Verkasalo PK, Iliadou A, Kaprio J, Koskenvuo $\mathrm{M}$, et al. Environmental and heritable factors in the causation of cancer-analyses of cohorts of twins from Sweden, Denmark, and Finland. N Engl J Med. 2000; 13;343(2): 78-85.

6. Holly JM, Perks CM. Cancer as an endocrine problem. Best Pract Res Clin Endocrinol Metab. 2008; 22(4): 539-550.

7. Henderson BE, Feigelson HS. Hormonal carcinogenesis. Carcinogenesis 2000; 21(3): 427-433.

8. Extermann M. Interaction between comorbidity and cancer. Cancer Control. 2007; 14(1): 13-22.

9. Karbownik-Lewinska M, Gesing A, Zasada K, Jedrzejczyk M, Sobieszczanska-Jablonska A, Krawczyk J, et al. Relationship between lipid peroxidation or carcinoembryonic antigen and risk factors for non-communicable diseases in women at midlife and beyond. Neuro Endocrinol Lett. 2012; 33(5): 536-545.

10. Karbownik-Lewinska M, Szosland J, Kokoszko-Bilska A, Stępniak J, Zasada K, Gesing A, et al. Direct contribution of obesity to oxidative damage to macromolecules. Neuro Endocrinol Lett. 2012; 33(4): 453-461.

11. Calle EE, Rodriguez C, Walker-Thurmond K, Thun MJ. Overweight, obesity, and mortality from cancer in a prospectively studied cohort of U.S. adults. N Engl J Med. 2003;348(17): 1625-1638.

12. King MC, Marks JH, Mandell JB. Breast and ovarian cancer risks due to inherited mutations in BRCA1 and BRCA2. Science 2003; 302(5645): 643-646.

13. Carmichael AR, Bates T. Obesity and breast cancer: a review of the literature. Breast. 2004; 13(2): 85-92.

14. Weiss HA, Brinton LA, Potischman NA, Brogan D, Coates RJ, Gammon $\mathrm{MD}$, et al. Breast cancer risk in young women and history of selected medical conditions. Int J Epidemiol. 1999; 28(5): 816-823.

15. Coughlin SS, Calle EE, Teras LR, Petrelli J, Thun MJ. Diabetes mellitus as a predictor of cancer mortality in a large cohort of US adults. Am J Epidemiol. 2004; 159(12): 1160-1167.

16. Szychta P, Szychta W, Gesing A, Lewiński A, Karbownik-Lewińska $\mathrm{M}$. TSH receptor antibodies have predictive value for breast cancer retrospective analysis. Thyroid Res. 2013; 6(1): 1-8.

17. Cristofanilli M, Yamamura Y, Kau SW, Bevers T, Strom S, Patangan M, et al. Thyroid hormone and breast carcinoma. Primary hypothyroidism is associated with a reduced incidence of primary breast carcinoma. Cancer. 2005;103(6): 1122-1128.

18. Kuijpens JL, Janssen-Heijnen ML, Lemmens VE, Haak HR, Heijckmann AC, Coebergh JW. Comorbidity in newly diagnosed thyroid cancer patients: a population-based study on prevalence and the impact on treatment and survival. Clin Endocrinol (Oxf). 2006; 64(4): 450-455.

19. Zadrożna A. [Raport $\mathrm{z}$ ogólnopolskiego badania ankietowego na temat postaw wobec palenia tytoniu] [In Polish]. TNS OBOP. 2011. http://www.gis.gov.pl/ckfinder/userfiles/files/PZ/Raport $\% 20$ og\%C3\%B3lnopolski\%20\%202011.pdf (access: 2014.04.05).

20. Lambe M, Hall P, Granath F, Sadr Azodi O, Nilsson T. Coronary angioplasty and cancer risk: a population-based cohort study in Sweden. Cardiovasc Intervent Radiol. 2005; 28(1): 36-38.

21. Lun Y, Wu X, Xia Q, Han Y, Zhang X, Liu Z. Hashimoto's thyroiditis as a risk factor of papillary thyroid cancer may improve cancer prognosis. Otolaryngol Head Neck Surg. 2013; 148(3): 396-402.

22. Torino F, Barnabei A, Paragliola R, Baldelli R, Appetecchia M, Corsello SM. Thyroid dysfunction as an unintended side effect of anticancer drugs. Thyroid. 2013; 23(11): 1345-1366.

23. Plummer M, Herrero R, Franceschi S, Meijer CJ, Snijders P, Bosch FX, et al. Smoking and cervical cancer: pooled analysis of the IARC multi-centric case--control study. Cancer Causes Control. 2003; 14(9): $805-814$.

24. Vona-Davis L, Rose DP. Adipokines as endocrine, paracrine, and autocrine factors in breast cancer risk and progression. Endocr Relat Cancer. 2007; 14(2): 189-206.

25. Villeneuve PJ, Johnson KC, Kreiger N, Mao Y. Risk factors for prostate cancer: results from the Canadian National Enhanced Cancer Surveillance System. The Canadian Cancer Registries Epidemiology Research Group. Cancer Causes Control. 1999; 10(5): 355-367. 